\title{
OMAE2018-77135
}

\section{HYDRODYNAMICS AROUND A DEEP-DRAFT SEMI-SUBMERSIBLE WITH VARIOUS CORNER SHAPES}

\author{
Yibo Liang \\ Department of Naval Architecture, Ocean \& \\ Marine Engineering \\ University of Strathclyde \\ Glasgow, G4 0LZ, UK
}

\author{
Longbin Tao \\ Department of Naval Architecture, Ocean \& \\ Marine Engineering \\ University of Strathclyde \\ Glasgow, G4 0LZ, UK
}

\begin{abstract}
A numerical study on flow over a stationary deep-draft semisubmersible (DDS) with various corner shapes was carried out to investigate the corner shape effects on the overall hydrodynamics. Three models based on a typical DDS design with different corner shapes were numerically investigated under $45^{\circ}$ incidence. The present numerical model has been validated by an experimental test carried out in a circulating water channel. It is demonstrated that, as the corner shape design changed, the hydrodynamic characteristics alter drastically. In addition, the flow patterns were examined to reveal some insights of the fluid physics due to the changing of different corner shape designs. The detailed numerical results from the parametric and geometric study will provide a good guidance for future practical designs.
\end{abstract}

\section{INTRODUCTION}

Along with the continuing developments in the field of offshore technology, an increasing number of deep-draft floating structures have been fabricated and installed in different deepwater regions around the world. Due to the increase of the submerged area, a deep-draft floating structure is subject to higher in-line and transverse hydrodynamic forces in the current which may induce stronger vortex shedding and lead to large amplitude vortex-induced motions.

Some of the deep-draft floating structures (such as TLP or semi-submersibles) consist of four rectangular columns. The hydrodynamics characteristics of bluff columns are largely depend on the behaviour of the shear layers that separate from the leading edge [1]. Thus, the hydrodynamic loading can be changed by controlling the separated flow. It is well know that drag forces on a square cylinder are reduced by rounding the corner [2]. Form this viewpoint, the present study is aimed to investigate the corner shape effects on the overall hydrodynamics around multi-columns structures to determine the fluid mechanism of the reduction in hydrodynamic loads on a DDS.

A large number of studies have been carried out to understand the effect of corner shape designs, such as Delany and Sorensen [3], Bearman, Graham, Obasaju and Drossopoulos [2], Tamura, Miyagi and Kitagishi [4], Tamura and Miyagi [1] as well as $\mathrm{Hu}$, Zhou and Dalton [5]. Most of the corner shape studies were focused on the 0 degree incidence. However, for square section shaped multi-columns structures, the most severe transverse motion which was induced by vortex shedding occurred at 45 degree incidence which was confirmed in both filed measurements and model tests [6-12]. Thus, the present numerical investigation about corner shape effects is focused on 45 degree incidence.

With the aim to study the hydrodynamics around a deepdraft semi-submersible with various corner shapes. Three different corner shape design (sharp, chamfered and rounded) are covered to compare the difference among them. This paper is mainly discusses the hydrodynamic loads on the structure as well as the flow patterns around the structure to reveal some insights of the fluid physics due to the changing of different corner shape designs.

\section{NOMENCLATURE}

$\begin{array}{ll}A & \text { Projected area } \\ B_{L} & \text { Platform width } \\ B_{T} & \text { Platform draft } \\ C_{D} & \text { Drag Coefficient } \\ C_{L} & \text { Lift Coefficient } \\ D & \text { Projected width of the column } \\ f & \text { Vortex shedding frequency }\end{array}$




$\begin{array}{ll}F_{D} & \begin{array}{l}\text { Hydrodynamic drag force acting on the } \\ \text { structure } \\ F_{L}, F_{y}\end{array} \quad \begin{array}{l}\text { Hydrodynamic lift force acting on the } \\ \text { structure } \\ F r\end{array} \quad \begin{array}{l}\text { Froude number } \\ H\end{array} \quad \text { Immersed length of the column } \\ P & \text { Pontoons height } \\ R e & \text { Reynolds number } \\ r m s & \text { Root Mean Square } \\ S & \text { Distance between two columns } \\ S t & \text { Strouhal number } \\ U, \quad U_{C} & \text { Free stream velocity } \\ \rho & \text { Fresh water density } \\ \omega & \text { Spanwise vorticity } \\ X & \text { In-line direction } \\ Y & \text { Transverse direction } \\ y^{+} & \text {Y plus value }\end{array}$

\section{MODEL CHRACTERISTICS}

The characteristics of benchmark DDS simulated in the current study are presented in Table 1 and Figure 1 . The model is built based on a prototype DDS design with a 1:128 scale ratio.

Different section shapes of the column have been simulated based on the benchmark model. Figure 2 shows column sectional configurations in the current simulations. The Reynolds number for computational is basically set to $4.3 \times 10^{4}$.
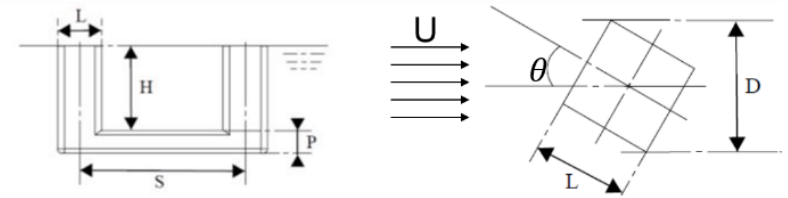

Figure 1 Characteristic dimensions of a DDS.

Table 1 Benchmark model characteristics.

\begin{tabular}{|c|c|}
\hline & Model (m) \\
\hline Distance between centre columns $(S)$ & 0.567 \\
\hline Column width $(L)$ & 0.152 \\
\hline $\begin{array}{l}\text { Immersed column height above the } \\
\text { pontoon }(H)\end{array}$ & 0.289 \\
\hline Pontoon height $(P)$ & 0.078 \\
\hline
\end{tabular}

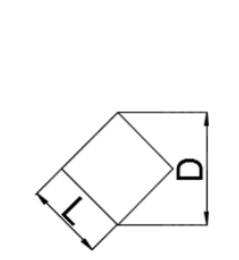

Sharp corners (benchmark model)

Figure 2 Column sectional configurations of a benchmark

\section{COPUTATIONAL OVERVIEW}

The detached eddy simulation (DES) was used in this study. For the DES model, the improved delayed detach eddy simulation (IDDES) model [13] with the Spalart-Almaras (SA) [14] was used. The delayed detached eddy simulation (DDES) [15] is a recently developed modification of the earlier established detached eddy simulation (DES) [14]. IDDES is a capable model which builds a single set of formulas both for natural (D)DES applications and for the wall-modelling in large eddy simulation (WMLES) [13]. The DDES length scale is implemented to eliminate the modelled-stress depletion in the original DES approach, while WMLES is applied to achieve more accurate prediction of the mean velocity in the boundarylayer. The boundary layers and irrotational regions are solved using SA model. However, when the grid is fine enough, it will emulate a basic large eddy simulation (LES) subgrid scale model in the detached flow regions [16]. This approach can improve the boundary layer simulation and in the meantime, reduce the computational cost. It is noted that the SA model requires $y^{+}<$ 1 (where $y^{+}=u * \Delta y_{1} / v$, and where $u_{*}$ denotes the friction velocity at the nearest wall, $\Delta y_{1}$ is the first layer thickness and $v$ is the kinematic viscosity) indicating that the viscous sublayer is properly resolved. All the simulations were carried out using a commercial CFD code, STAR-CCM+ 9. The finite volume method (FVM) is adopted to discretize the incompressible flow field equations [17]. The second-order implicit three time levels (ITTL) scheme is applied for the temporal discretization. The convective term is evaluated by using a hybrid second-order upwind scheme. SIMPLE algorithm is employed to treat the pressure and velocity coupling.

\section{Governing equations}

The governing Navier-Stokes equations solved for the incompressible flow can be written as:

$$
\begin{aligned}
& \nabla \cdot \bar{u}=0, \\
& \frac{\partial \bar{u}}{\partial \bar{t}}+\bar{u} \cdot \nabla \cdot \bar{u}=-\frac{1}{\rho} \nabla \bar{p}+v \nabla^{2} \bar{u}+\frac{1}{\rho \nabla \tau},
\end{aligned}
$$

where $\nabla$ is the Hamiltonian operator; $u$ is the velocity vector; $t$ is the time; $p$ is the pressure; $\rho$ is the density of water; $v$ is the kinematic viscosity of the water; The last term of Equitation (2) is the Reynolds stress tensor $\tau=-\rho\left(\overline{u^{\prime} u^{\prime}}\right)$, where $u^{\prime}$ denotes the fluctuating velocity. The Reynolds stress tensor is an additional term that represents the effects of turbulence.

\section{Computational domain}

For all of the simulations, a $9 B_{L} \times 6 B_{L} \times 3 B_{T}$ sized computational domain was used in the present simulations (where $B_{L}$ is the overall width of the structure and $B_{T}$ is the draft of the structure). Zou, Lin and Lam [18] previously used a $32 L \times 20 L \times 3 L$ (about $7.1 B_{L} \times 4.4 B_{L} \times 3 L$ ) domain. The computational domains were $6 B_{L} \times 4.5 B_{L} \times 2.8 B_{T}$ and $5 B_{L} \times 4 B_{L} \times 2.2 B_{T}$ in the studies by Lee, Chien and Gu [11]. Tan, Magee, Kim, Teng and Zukni [19] performed a $27 B_{L} \times 18 B_{L} \times 6.5 B_{T}$ domain and Liu, Xiao, Lyu and Tao [20] used a $11 B_{L} \times 6 B_{L} \times 3 B_{T}$ domain. Koop, Rijken, Vaz, Maximiano and Rosetti [12], however, chose a $10 B_{L} \times 6 B_{T}$ cylindrical domain for simulating flow with their numerical 
models. In the earlier study [21], a computational domain size of $9.5 B_{L} \times 6.3 B_{L} \times 3 B_{T}$ was employed. Compared with aforementioned computational domain sizes, a $9 B_{L} \times 6 B_{L} \times 3 B_{T}$ domain (see Figure 3 ) was considered to be sufficiently large to eliminate both the far field effects from the boundaries and the three-dimensional effects from a spanwise cross flow direction.

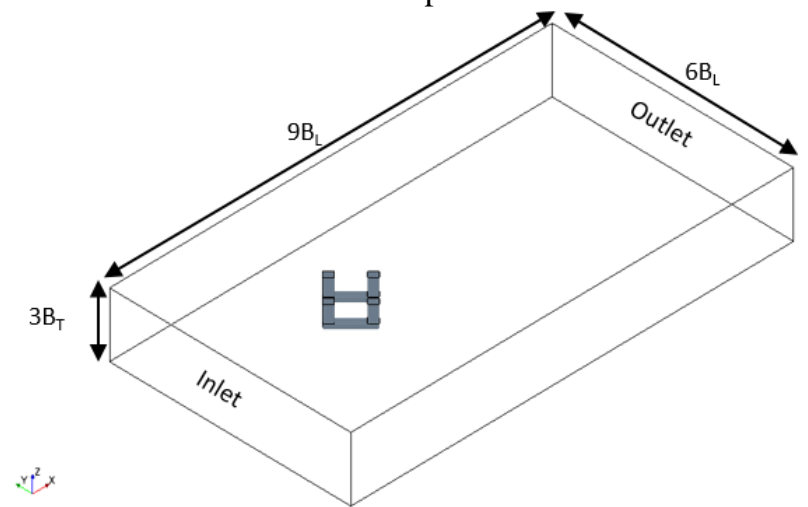

Figure 3 Computational domain.

The computational domain was modelled with a threedimensional mesh of elements. A polyhedral mesh [16] was used in this study. The overall element mesh domain is illustrated at a mid-depth horizontal layer in Figure 4. In the present study, a near wall refinement method named "Prism Layer Mesher [16]" was adopted. The "Prism Layer Mesher" model (as shown in Figure 5) is used with a core volume mesh to generate orthogonal prismatic cells next to wall surfaces. This layer of cells is necessary to improve the accuracy of the flow solution [16]. The $\mathrm{y}^{+}$values were smaller than 1 in all simulations to improve the performance of the boundary layer simulation. Another five regional refinements were added in the domain in order to refine both the near wake and the far wake regions (see Figure 6).

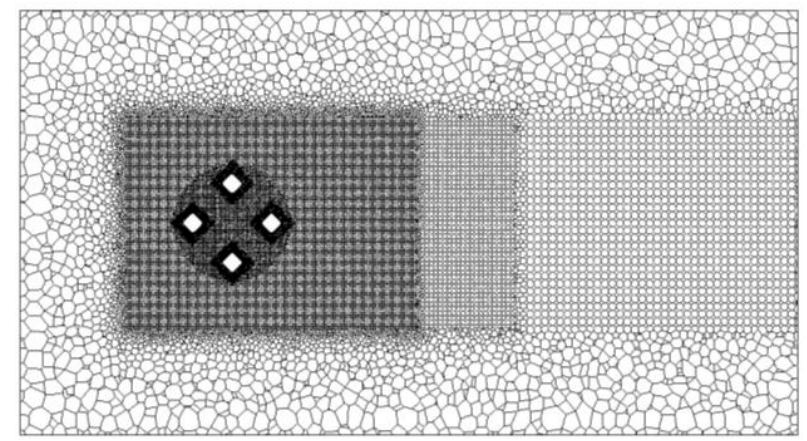

Figure 4 Visualization of the mesh at the middle draft level of the DDS ( $X Y$ plane at the middle draft of the DDS).

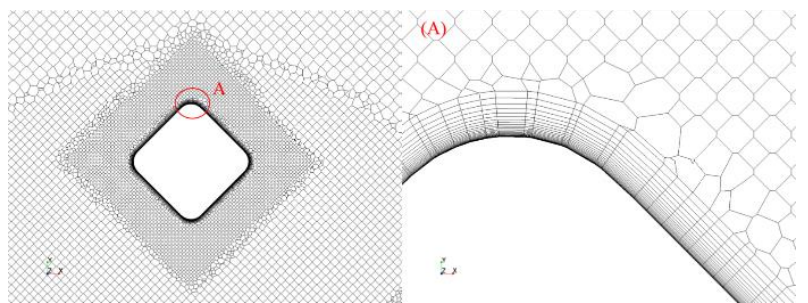

Figure 5 Mesh around the column at the middle draft level of the DDS ( $X Y$ plane at the middle draft of the DDS) showing the "Prims Layer Mesher".

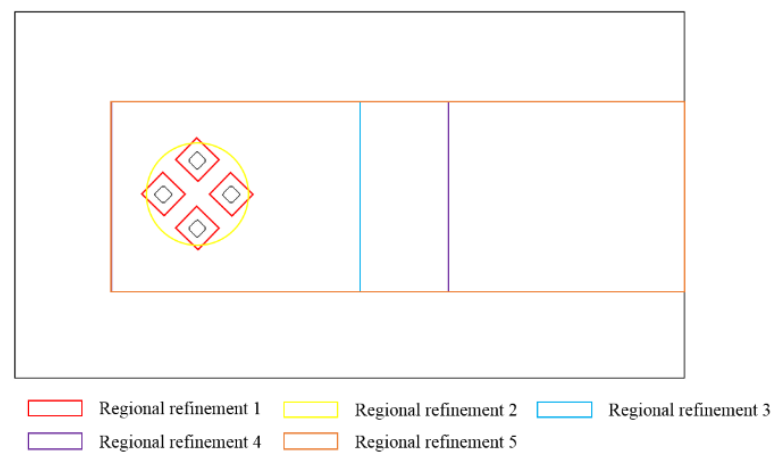

Figure 6 The outlines of five regional refinements.

The boundary conditions are kept same in all the simulations. At the inlet, a uniform and constant flow velocity is specified directly for all sensitivity studies. Along the outlet boundary, the pressure is prescribed to be equal to zero. The velocity at the boundary is extrapolated from the interior using reconstruction gradients [16]. For the body surface of the DDS, a no-slip boundary condition is specified in terms of the tangential velocity which is explicitly set to be zero and the pressure at the boundary is extrapolated from the adjacent cells using reconstruction gradients [16]. It is noted that the Froude number is quite small $(F r<0.2, F r=U / \sqrt{g D}$, where $U$ is the current velocity, $g$ is the acceleration of gravity and $D$ is the projected width of the column) in all simulations of the present investigation. As observed in the physical model tests [22], the free surface effects were rather limited and can be ignored. Therefore, only the submerged geometry is considered, and the geometry of the structure above the waterline will not affect the simulation results.

\section{Sensitivity and Validation Study}

The details of mesh and time step sensitivity studies have been presented in the previous studies $[22,23]$. In the present study, the results for all cases were obtained by averaging after more than fifty vortex shedding cycles.

The present numerical predictions have been validated by the experiment carried out in a circulating water channel at Shanghai Jiao Tong University. A comparison between the numerical and experimental results at $R e=4.3 \times 10^{4}$ has been illustrated in Table 2. The numerical results show a good agreement compared with the experimental results. 
In Table 2 , the force coefficients $\left(C_{D}, C_{L}\right)$ are the nominal coefficients describing the drag and lift forces on the structure, which are defined as:

$$
\begin{gathered}
C_{D}=\frac{F_{D}}{\frac{1}{2} \rho U_{C}^{2} A}, \\
C_{L}=\frac{F_{L}}{\frac{1}{2} \rho U_{C}^{2} A},
\end{gathered}
$$

where, $F_{D}$ is the drag force on the structure, $F_{L}$ is the lift force on the structure, $\rho$ is the fresh water density, $U_{C}$ is the free stream velocity, $A$ is the projected area of the immersed structure.

The Strouhal number $(S t)$ is a dimensionless number describing oscillating flow mechanisms (e.g. vortex shedding phenomenon) which is given by:

$$
S t=\frac{f D}{U_{c}}
$$

where $f$ is the vortex shedding frequency that is obtained from the power spectra of lift force coefficient fluctuations as followed by Schewe [24] and $D$ is the projected width of the column.

Table 2 Comparison of the results from the present numerical calculations and experimental measurements at $R e=$ $4.3 \times 10^{4}$ for rounded corner case.

\begin{tabular}{clll}
\hline & Numerical & Experimental & Relative variation (\%) \\
\hline$\overline{\boldsymbol{C}}_{\boldsymbol{D}}$ & 1.119 & 0.973 & $15.01 \%$ \\
$\boldsymbol{C}_{\boldsymbol{L r m s}}$ & 0.077 & 0.073 & $5.47 \%$ \\
$\boldsymbol{S} \boldsymbol{t}$ & 0.191 & 0.173 & $10.40 \%$ \\
\hline
\end{tabular}

\section{RESULTS AND ANALYSIS}

Three different corner shapes (sharp, chamfered and rounded) of a typical deep-draft semi-submersible under 45 degree flow incidence have been numerically investigated after present and previous rigorous validations [21-23] against the experimental data. The hydrodynamic loads on different members of the structure, such as four columns and pontoons, are compared in order to quantify the corner shape effects. In addition, the flow patterns are further examined to reveal some insights of the vortex dynamics around the DDS.

\section{Overall drag and lift forces}

The overall drag and lift forces are presented as the nondimensional force coefficients $C_{D}, C_{L}$ and St. Details of the numerical results are shown in Figure 7, Figure 8 and Figure 9.

As can be seen in Figure 7, unlike the previous study at 0 degree incidence $[1,4]$, the chamfered coroner design does not affect the drag coefficient on the overall structure. It is due to the projected area of chamfered corner shape design at 45 degree is slightly smaller than the projected area of the sharp corner shape design. However, by changing the corner shape to rounded corner, both drag coefficient is decreased slightly. Additionally, the vortex shedding frequency is dropped when the corner shape has been modified (see Figure 9).

When it comes to the lift coefficient, the trend of the corner shape effects is changed. Both chamfered and rounded corner designs have a larger lift coefficient compared with the sharp corner design (see Figure 8). And the chamfered corner design has the largest lift coefficient among all three corner shapes.

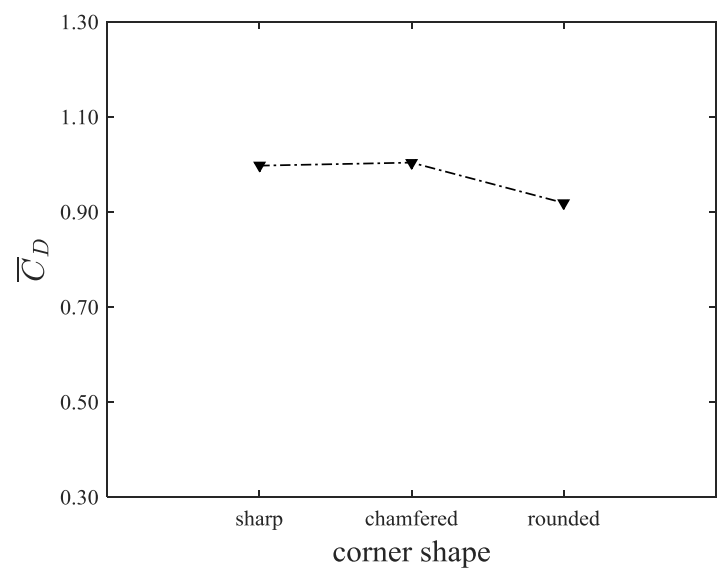

Figure 7 Mean drag coefficient $\left(\bar{C}_{D}\right)$ from the numerical results with various corner shapes.

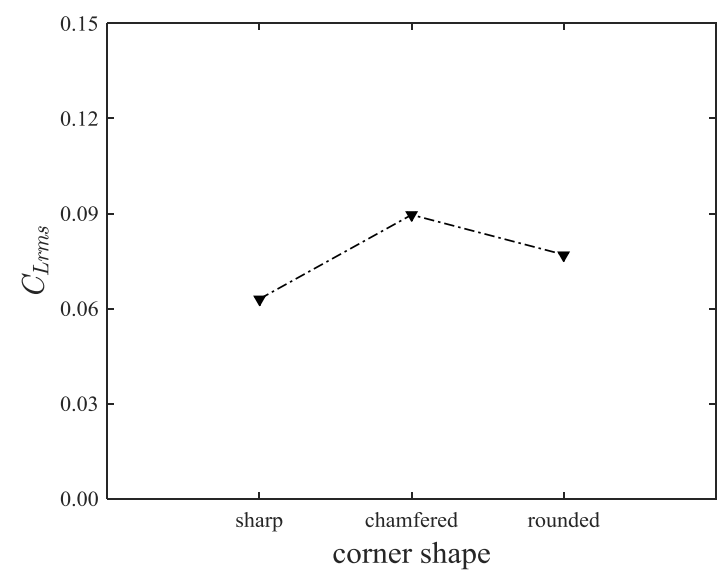

Figure 8 Root mean square lift coefficient $\left(C_{\text {Lrms }}\right)$ from the numerical results with various corner shapes.

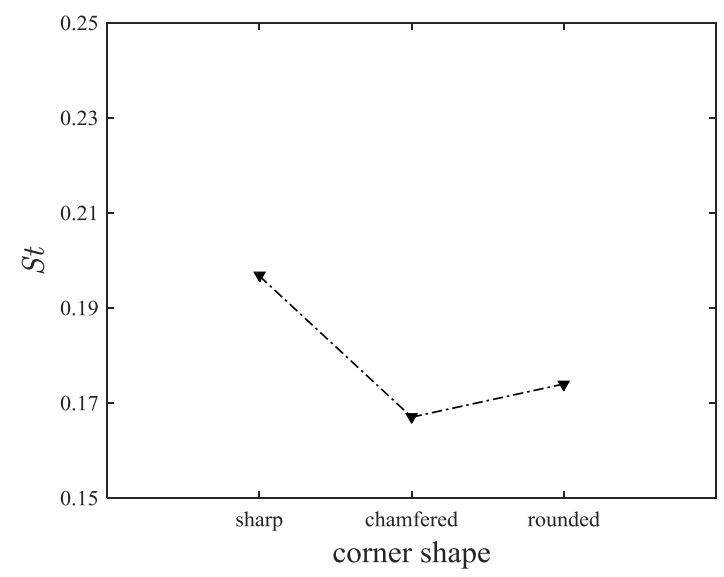

Figure 9 Strouhal number $(S t)$ from the numerical results with various corner shapes. 


\section{Drag and lift forces on each member of the DDS}

In order to improve the understanding of interactions between vortex shedding processes due to each structures member of the DDS, the drag and lift forces on each member of the DDS are calculated and presented in Figure 10 and Figure 11. The column definition is defined in Figure 12.

For the mean drag coefficients, as can be seen in Figure 10, most of the members follow the same trend as the overall structure by changing the corner shape. However, the upstream column (column 1) shows a slightly different trend. The $\bar{C}_{D}$ on the column 1 is increased when it changed to chamfered corner and decreased when it changed to rounded corner. However, for the root-mean-square lift coefficient, as shown in Figure 11, the downstream column (column 3) shows a different trend compared with other columns. The chamfered corner design does not have a strong effect on the column 3 . It can be clearly seen in Figure 10 and Figure 11, the two side columns (column 2 and 4) are significant affected by various coroner shape designs. Due to the pontoons were kept same for three different models. The hydrodynamic forces on the pontoons are remain stable among all three models.

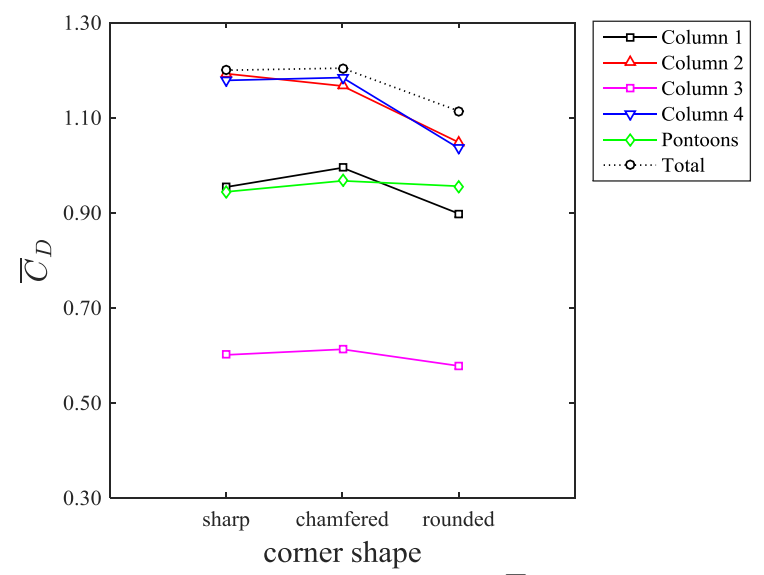

Figure 10 Mean drag coefficient $\left(\bar{C}_{D}\right)$ on each member of the DDS with various corner shapes.

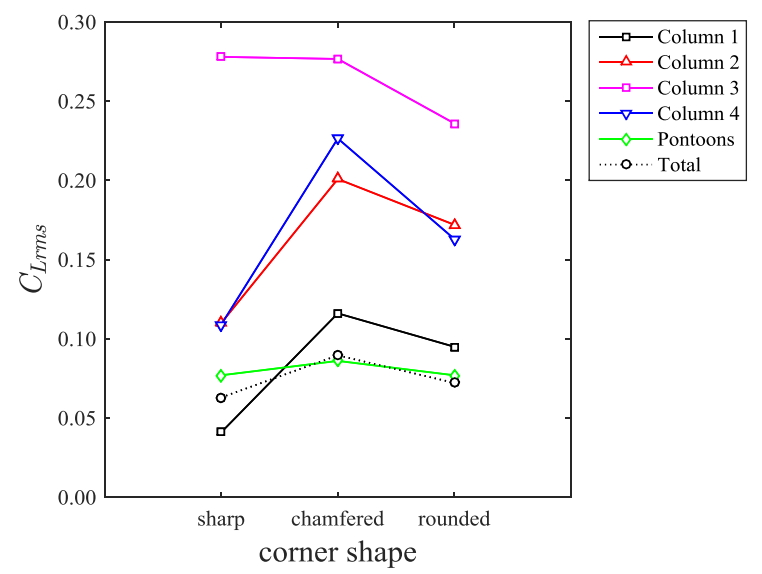

Figure 11 Root-mean-square lift coefficient $\left(C_{\text {Lrms }}\right)$ on each member of the DDS with various corner shapes.
A)

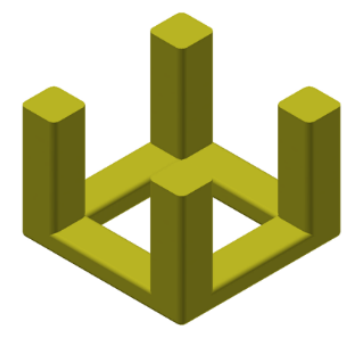

B)

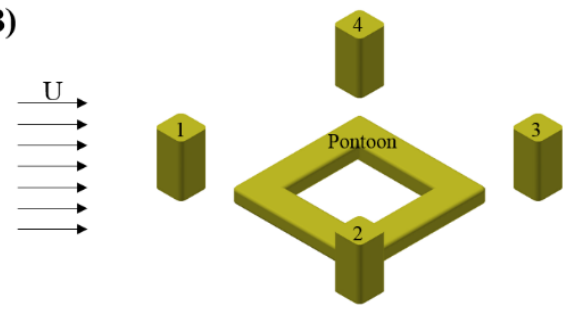

Figure 12 Column definition of the DDS model

\section{Flow patterns}

In order to have a general visual appreciation of the vortex shedding patterns, the vorticity contours are plotted in Figure 13. The non-dimensional spanwise vorticity is used to describe the spanwise vorticity in the current study, which is defined as:

non-dimensional spanwise vorticity $=\omega D / U$,

where $\omega$ is the $z$ components of the vorticity, $D$ is the projected length of the column and $U$ is the current speed.

As can be seen in Figure 13, by changing the corner shapes, the vortex shedding streets after columns have been slightly narrow down (on Y/in-line direction). In addition, the timeaveraged velocity distribution around the DDS has been plotted to show the difference among three different column designs. It also shows that, the chamfered and rounded corner designs narrow down the vortex shedding streets (on Y/in-line direction) after each column. Moreover, for the two side columns (column 2 and 4) as well as the downstream column (column 3), the recirculation regions (on $X /$ transverse direction) are shorten by changing the corner shape to chamfered and rounded corners. Additionally, the recirculation zone after the column 3 for the rounded corner shape design has a flat velocity contour lines and is smaller than the chamfered corner shape design.

\section{CONCLUSIONS}

The hydrodynamics around a deep-draft semi-submersible with various corner shapes at 45 degree incidence have been numerically studied in the present work. The major results are summarized as:

(1) For 45 degree incidence, the rounded corner design decreases the drag coefficient and vortex shedding frequency of the overall structure compared with the sharp corner design. Both chamfered and rounded corner designs can increase the lift coefficient on the overall structure compared with the sharp corner design. In addition, the chamfered corner design has a larger increase of the lift coefficient than the rounded corner design. 
(2) The two side columns (column 2 and 4) are significantly affected by the corner shape design compared with the upstream column (column 1) and the downstream column (column 3).

(3) By changing the corner shapes (from sharp corner to chamfered/rounded corner), the vortex shedding streets after columns have been slightly narrow down for both in-line and transverse direction. Additionally, the recirculation region after column 3 for the rounded corner design has a flat velocity contour lines which is different as the sharp and chamfered corner design.

\section{ACKNOWLEDGEMENTS}

The authors would like to acknowledge the support of Newton Fund of Royal Academy of Engineering UK (NRCP/1415/211) and the National Natural Science Foundation of China (Grant No.51279104). This work made use of the facilities of N8 HPC Centre of Excellence, provided and funded by the N8 consortium and EPSRC (Grant No.EP/K000225/1).

\section{REFERENCES}

[1] Tamura, T., and Miyagi, T., 1999, "The effect of turbulence on aerodynamic forces on a square cylinder with various corner shapes," Journal of Wind Engineering and Industrial Aerodynamics, 83(1), pp. 135-145.

[2] Bearman, P. W., Graham, J. M. R., Obasaju, E. D., and Drossopoulos, G. M., 1984, "The influence of corner radius on the forces experienced by cylindrical bluff bodies in oscillatory flow," Applied Ocean Research, 6(2), pp. 83-89.

[3] Delany, N. K., and Sorensen, N. E., 1953, Low-speed drag of cylinders of various shapes, National Advisory Committee for Aeronautics.

[4] Tamura, T., Miyagi, T., and Kitagishi, T., 1998, "Numerical prediction of unsteady pressures on a square cylinder with various corner shapes," Journal of Wind Engineering and Industrial Aerodynamics, 74, pp. 531-542.

[5] Hu, J. C., Zhou, Y., and Dalton, C., 2005, "Effects of the corner radius on the near wake of a square prism," Experiments in Fluids, 40(1), pp. 106-118.

[6] Waals, O. J., Phadke, A. C., and Bultema, S., 2007, "Flow Induced Motions on Multi Column Floaters," ASME 2007 26th International Conference on Offshore Mechanics and Arctic Engineering, American Society of Mechanical Engineers, pp. 669-678.

[7] Rijken, O., and Leverette, S., 2008, "Experimental Study into Vortex Induced Motion Response of Semi Submersibles with Square Columns," ASME 2008 27th International Conference on Offshore Mechanics and Arctic Engineering, American Society of Mechanical Engineers, pp. 263-276.

[8] Magee, A., Sheikh, R., Guan, K. Y. H., Choo, J. T. H., Malik, A. M. A., Ghani, M. P. A., and Abyn, H., 2011, "Model tests for VIM of multi-column floating platforms," ASME 2011 30th International Conference on Ocean, Offshore and Arctic Engineering, American Society of Mechanical Engineers, pp. 127-136.
[9] Gonçalves, R. T., Rosetti, G. F., Fujarra, A. L. C., and Oliveira, A. C., 2012, "Experimental study on vortex-induced motions of a semi-submersible platform with four square columns, Part I: Effects of current incidence angle and hull appendages," Ocean Engineering, 54, pp. 150-169.

[10] Ma, W., Wu, G., Thompson, H., Prislin, I., and Maroju, S., 2013, "Vortex induced motions of a column stabilized floater," Proceedings of the DOT International Conference, pp. 22-24.

[11] Lee, S.-K., Chien, H.-P., and Gu, H., 2014, "CFD Study of Deep Draft SemiSubmersible VIM," Offshore Technology Conference-Asia, Offshore Technology Conference.

[12] Koop, A., Rijken, O., Vaz, G., Maximiano, A., and Rosetti, G., 2016, "CFD Investigation on Scale and Damping Effects for Vortex Induced Motions of a Semi-Submersible Floater," Offshore Technology Conference, Offshore Technology Conference.

[13] Shur, M. L., Spalart, P. R., Strelets, M. K., and Travin, A. K., 2008, "A hybrid RANS-LES approach with delayed-DES and wall-modelled LES capabilities," International Journal of Heat and Fluid Flow, 29(6), pp. 1638-1649.

[14] Spalart, P. R., Jou, W. H., Strelets, M., and Allmaras, S. R., 1997, "Comments on the feasibility of LES for wings, and on a hybrid RANS/LES approach," Advances in DNS/LES, 1, pp. 48.

[15] Spalart, P. R., Deck, S., Shur, M. L., Squires, K. D., Strelets, M. K., and Travin, A., 2006, "A new version of detached-eddy simulation, resistant to ambiguous grid densities," Theoretical and Computational Fluid Dynamics, 20(3), pp. 181-195.

[16] CD-adapco, 2014, "User Guide," Star-CCM+ Version 9.04. [17] Blazek, J., 2015, Computational fluid dynamics: principles and applications, Butterworth-Heinemann.

[18] Zou, L., Lin, Y.-f., and Lam, K., 2008, "Large-eddy simulation of flow around cylinder arrays at a subcritical Reynolds number," Journal of Hydrodynamics, Ser. B, 20(4), pp. 403-413.

[19] Tan, J. H. C., Magee, A., Kim, J. W., Teng, Y. J., and Zukni, N. A., 2013, "CFD Simulation for Vortex Induced Motions of a Multi-Column Floating Platform," ASME 2013 32nd International Conference on Ocean, Offshore and Arctic Engineering, American Society of Mechanical Engineers, pp. V007T008A066-V007T008A066.

[20] Liu, M., Xiao, L., Lyu, H., and Tao, L., 2015, "Numerical Analysis of Pontoon Effect on Flow-Induced Forces of the Deep Draft Semisubmersible in a Cross-Flow," ASME 2015 34th International Conference on Ocean, Offshore and Arctic Engineering, American Society of Mechanical Engineers, pp. V001T001A030-V001T001A030.

[21] Liang, Y., Tao, L., Xiao, L., and Liu, M., 2016, "Experimental and Numerical Study on Flow Past Four Rectangular Columns in Diamond Configuration," ASME 2016 35th International Conference on Ocean, Offshore and Arctic Engineering, American Society of Mechanical Engineers, pp. V001T001A044-V001T001A044.

[22] Liang, Y., and Tao, L., 2017, "Interaction of vortex shedding processes on flow over a deep-draft semi-submersible," Ocean Engineering, 141, pp. 427-449. 
[23] Liang, Y., Tao, L., Xiao, L., and Liu, M., 2017, "Experimental and numerical study on vortex-induced motions of a deep-draft semi-submersible," Applied Ocean Research, 67, pp. 169-187.
[24] Schewe, G., 1983, "On the force fluctuations acting on a circular cylinder in crossflow from subcritical up to transcritical Reynolds numbers," Journal of Fluid Mechanics, 133, pp. 265285.
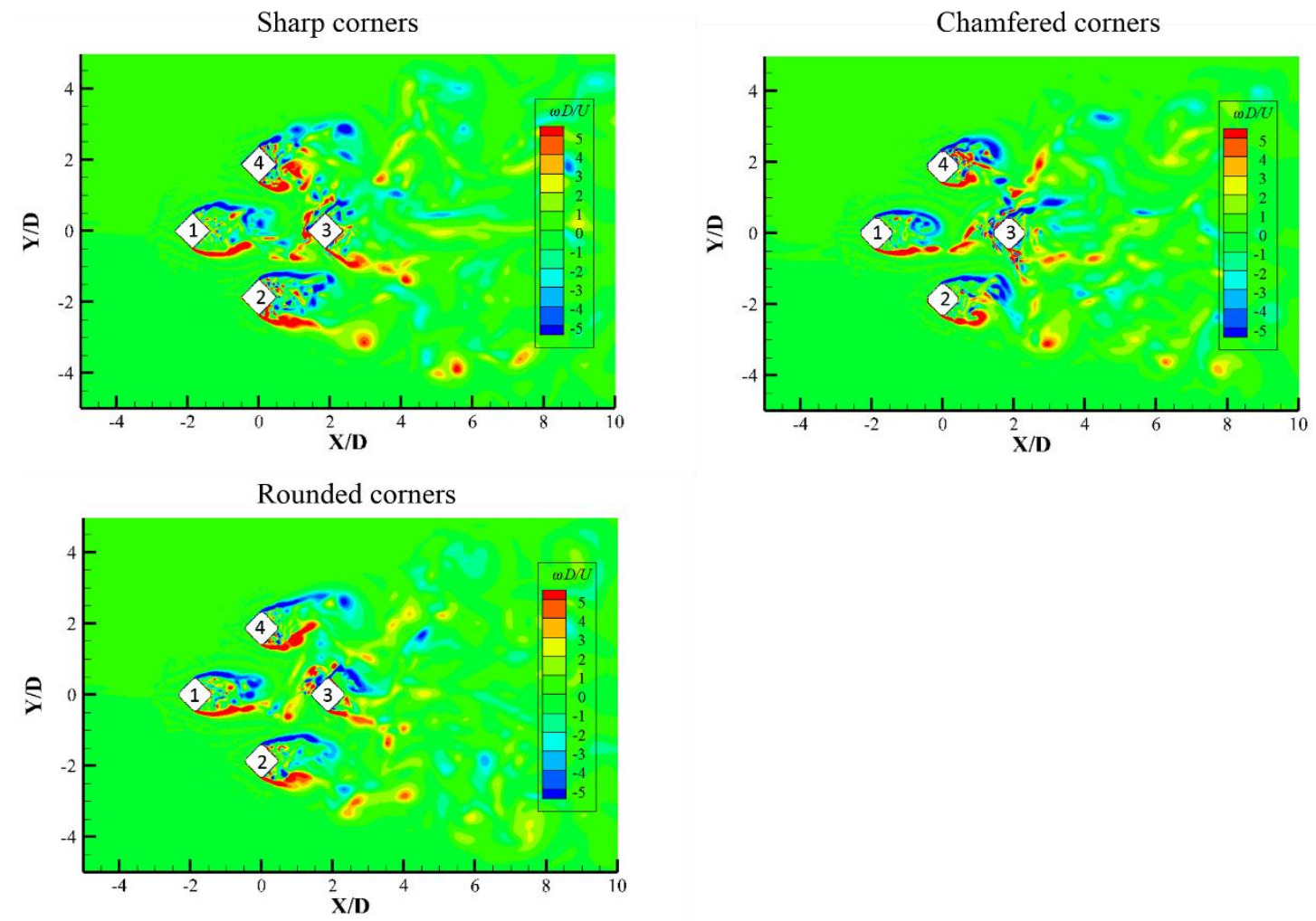

Figure 13 Non-dimensional spanwise vorticity contours with various corner shapes. 

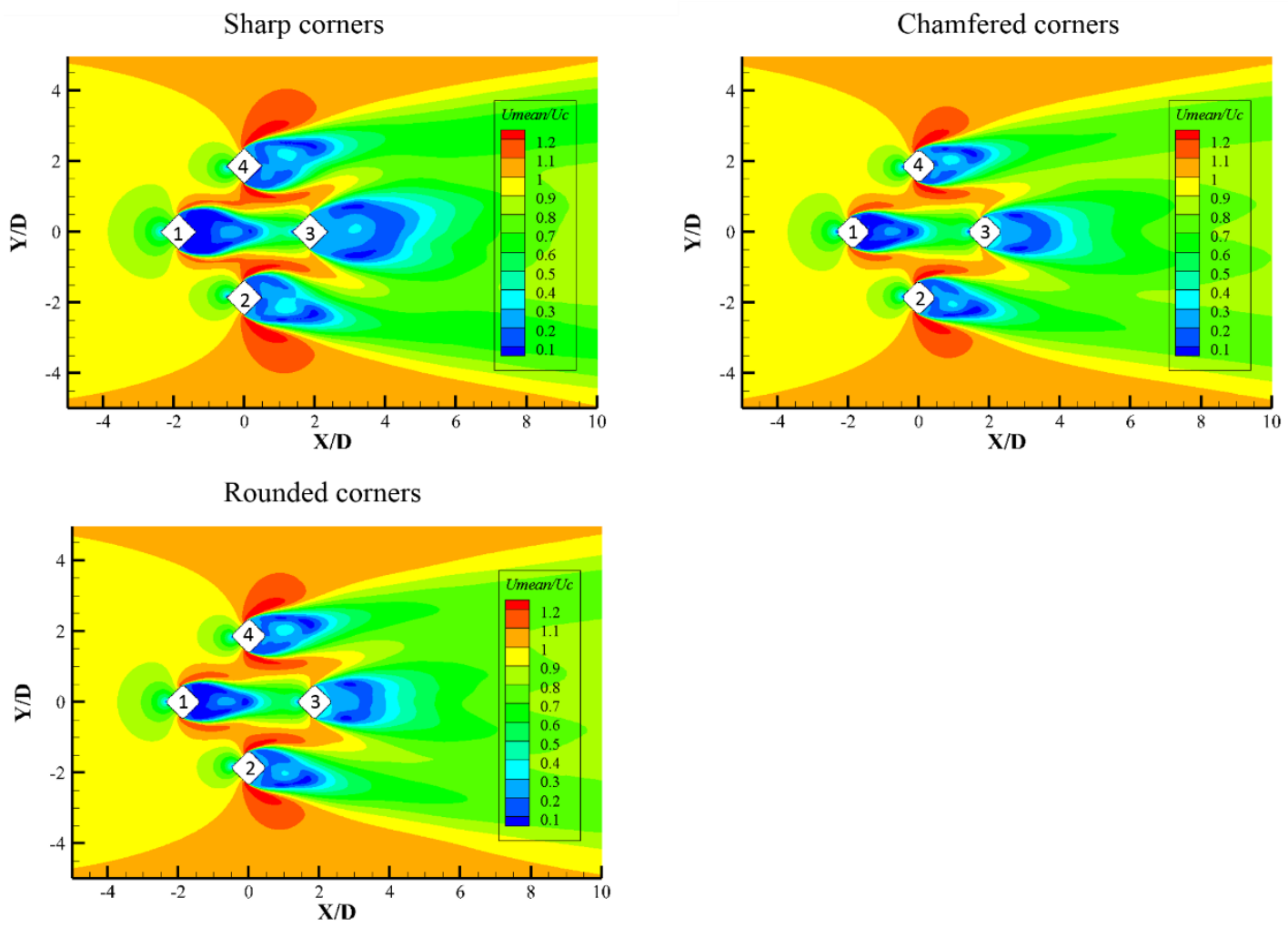

Figure 14 Time-averaged velocity distribution around the DDS with various corner shapes. 